Indian J. Phys. 67A (6), 571 - 576 (1993)

\title{
Phase-driven current in the quantum Hall geometry*
}

\author{
Vipin Srivastava \\ Cavendish Laboratory, Cambridge University, Cambridge CB3 OHE, UK, and \\ School of Physics. University of Hyderabad, Hyderabad.500 134, India ${ }^{\dagger}$
}

Received 26 May 1988

\begin{abstract}
We discuss the possiblity of flow of a transverse current driven by phase slip between the edge currents in a narrow quantum Hall sample. The underlying physics and the consequences appear exactly parallel to those of the Josephson tunnel junctions for instance, we can predict the appearance of Shapiro steps in $1 . V$ characteristics on application of a high. frequency AC voltage hetween the two edges, and the existence of a Riedel-type peak in the frequency dependence of the $\mathrm{AC}$ elc The analysis throws fresh light on the quantum interference phenomena currently being discussed extensively in connection with the quantum Hall effect.
\end{abstract}

Keywords : Quantum Hall effect, phase driven current.

PACS No. : 73 40.Hm.

Much new physics has been learnt as a consequence of the discovery of the quantum Hall effect (QHE) observed in two-dimensional disordered systems at high magnetic ficlds and low temperatures [1]. Here we point out that a Josephson-type effect should be present in narrow quantum Hall samples, i.e. a phase-driven alternating current (AC) should flow between the edges of the sample. This will give rise to an $\mathrm{AC}$ component in the net current flowing along the length of the sample. Imry and others [2] have earlier made suggestıons about the possible connection of the AC Josephson and quantum Hall effects under spocial circumstances. Imry, for instance, considers a potential that varies only along the direction of the sample current, say the $x$ direction, disregards its dependence on $y$ and takes the Hall voltage as induced by a time-varying Aharonov-Bohm (AB) flux flowing in the $x$ direction. We do not suggest any modification in the geometry apart from demanding that the width of the sample should be sufficiently small.

- Originally publised in J. Phys. C21 L815-L819 (1988) and is reproduced here with the permission received from IOP publishing Limited, U.K., dt. April 6, 1993. Keywords and PACS No. have only been incorporated here for retreival purpose.

$\uparrow$ Permanent address 
The role played by edge currents in causing the QHE is crucial (see [3] and references therein) and it is quite certain that a major fraction of the total current flows along the edges in narrow samples [4]. That the long-range order in the phase of the wavefunction (to ensure it is single valued) is significant for QHE [5] is also established beyond doubt. The long-range phase coherence, under the condition when the edge currents are near enough to be weakly coupled, can set up a transverse AC (in the $y$ direction); the weak coupling can be provided by certain equipotential regions percolating between the edges or by occasional overlaps of the wavefunctions of the current-carrying electrons that otherwise spread lengthwise close to the edges. To see this, we will first recall the role played by the phase coherence in yielding the $\mathrm{AB}$ effect (for a review see [6]) in resistive systems, and then use this knowledge to model a situation that can give rise to a Josephson-type effect in a conventional QHE experiment on narrow samples.

It has been settled unambiguously by the observation of the $\mathrm{AB}$ effect in resistive systems [6] that so long as the inelastic scattering is minimal, the electrons traverse coherently over macroscopic length scales, i.e. the wavefunction maintains its phase coherence all the way across the sample. If the electrons enter the device in phase, they are phase shifted relative to each other on reaching the other end because of elastic collisions with defects on the way, hut the phase memory is maintained along each path [7], and the current can be laken as a phase-coherent superposition of wavefunctions in all the paths. For each electron the phase changes in a coherent and delerministic manner, although in the end it takes a set of uncorrelated values for all electrons. The appearance of the $A B$ effect in such conditions implies that the parts of an electron wavefunction that pass through the two arms of the $A B$ ring, when recombined, may have undergone arbitrary changes in their respective phases, but at the end their relatıve phase difference must be $2 \pi$, and that this must happen for almost all electrons independently of each other.

Taking our cue from this remarkable behaviour exhibited by electrons in the resistive systems that preserve phase coherence, we propose the following for our system. Each current-carrying electron along an edge can be represented by a phase-coherent supcr-position of energy eigenstates, i.e. by a wavefunction of the type $C(r) \exp (\mathrm{i} \alpha)$. In the highly degenerate Landau system $C(r)$ can be normalised to be proportional to charge density per Landau sub-band along each edge. At a given magnetic field $B$, the $n$ electrons per Landau sub-band per unit area can be divided between the two edges as, say, $n_{1}$ and $n_{2}$ such that $n_{1}+n_{2}=n$. The charge density at the edges 1 and 2 will then be proportional to $n_{1}$ and $n_{2}$ respectively. Now if the sample is narrow and the edge currents fall so close to each other that they can be assumed to be weakly coupled, then the non-zero probability of electrons making transitions between the edge currents will demand that the wavefunction of an electron near the edge 1 includes in its 'phase-coherent superposition' certain unoccupied states of the same energy near the edge 2; and, as we will see, if the phase of these states (near the edge 2) is the same, or nearly the same, as that of the wavefunction on the edge 1 (before the coupling 
became non-zero) then the energy of the system will be lowered. All the electrons on either edge that can find unoccupied states of the same energy near the other edge into which they can make phase-coherent transition should do this individually and independently of each other. This process will he favoured hecause it minimises the total energy of the system, also the number-phase uncertainty relation suggests that the electron exchange will be appreciable if the phase change remains more or less zero in the course of the exchange. Wc may note in passing that all this ties up nicely with Anderson's ideas on broken symmetry and long-range phase order (for an interesting account see [8]) - it seems it is responsible for the unique property described above just as it is for,most of the unique propertics of superconductivity, superfluidity etc.

Representing the amplitude to find an electron near the edge 1 by $\psi_{1}$ and the amplitude to find it near the other by $\psi_{2}$ and the coupling conslant by $K$, we can solve the problem of leakage between the two edge currents (within the framework described above) like a "flipflop' problem of a two-state system. The following derivation runs parallel to that in the Josephson effect. but we include a simplified version (after Feynman [9]) for the sake of completeness. The $\psi_{1}$ and $\psi_{2}$ can be related by

$$
i \hbar \partial \psi_{a} / \partial t=U_{a} \psi_{a}+K \psi_{b}
$$

where $a, b \equiv \mathrm{I}, 2$ and $U_{a}$ is the energy at the edge $a$. With the $\psi$ as described above, and $U_{1}= \pm e V_{I I} / 2$ for the Hall voltage $V_{H}$ between the two cdges and the energy zero taken hetween $U_{1}$ and $U_{2}$, we obtain

$$
\begin{aligned}
& \partial n_{1} / \partial t=(2 / \hbar) K\left(n_{1} n_{2}\right)^{1 / 2} \sin \varphi \\
& \partial n_{2} / \partial t=-(2 / \hbar) K\left(n_{1} n_{2}\right)^{1 / 2} \sin \varphi
\end{aligned}
$$

and

$$
\begin{aligned}
& \partial \alpha_{1} / \partial t=-(K / \hbar)\left(n_{2} / n_{1}\right)^{1 / 2} \cos \varphi-\left(e V_{\mathrm{H}} / 2 \hbar\right) \\
& \partial \alpha_{2} / \partial t=-(K / \hbar)\left(n_{1} / n_{2}\right)^{1 / 2} \cos \varphi+\left(e V_{\mathrm{H}} / 2 \hbar\right)
\end{aligned}
$$

where $\varphi=\alpha_{2}-\alpha_{1}$. Since the current density should have its direction opposite to that of the phase gradient, from (2) we find that if $\varphi>0$, then electrons should flow from edge 1 to edge $2, K$ should be negative and the current densiy from edge 2 to edge 1 should be given by

$$
J=J_{c} \sin \varphi
$$

$J_{c}$ is the critical value of $J$ at $\varphi=\pi / 2$. From equations (3) we deduce that the coupling between $\psi_{1}$ and $\psi_{2}$ reduces the energy by

$$
E_{c}=\left(\pi J_{c} / e\right) \cos \varphi
$$


Thus, as the phase difference increases from 0 to $\pi / 2$, the current density from 2 to 1 increases. from zero to the maximum value $J_{c}$. Concurrently, the phase-coupling energy decreases from its maximum to zero. In a disordered medium the current has to find its way through a randomly fluctuating potential. The electrons can flow along equipotential paths percolating from one edge to the other ${ }^{\dagger}$, as well as by surmounting barriers of suitable height and width.

Since the Hall voltage is always non-zero for non-zero $B$, the relative phase slip continues to occur all the time and is given from equation (3) by

$$
\partial \varphi / \partial t=e V_{\mathrm{H}} / \hbar \quad \text { (assuming } n_{1}=n_{2} \text { ). }
$$

Consequently a high-frequency $\mathrm{AC}$ is flowing in the system with zero net current on average. The equations (4) and (6) are exactly the same as the Josephson equations [10], but pertain to single electrons. To observe the $\mathrm{AC}$ in an experiment either a single cycle of the AC (a single current loop) should somehow be tapped and made to show its effect, or the AC made to resonate with an external frequency source. The former, although apparently more difficult, may prove important for quantum interference studies as explained in a forthcoming paper. The latter, which is a more direct method, can be performed by adding an $\mathrm{AC}$ component to the existing DC Hall voltage, i.e. hy applying a time-dependent voltage,

$$
V(t)=V_{\mathrm{H}}+v \cos \omega t \quad\left(v \ll V_{\mathrm{H}}\right) .
$$

Then from equation (6),

$$
\varphi(t)=\varphi_{0}+\left(e V_{\mathrm{H}} / \hbar\right) t+(e v / \hbar \omega) \sin \omega \quad \varphi_{0} \equiv \varphi(t=0)
$$

which when inserted in equation (4) gives

$$
J=J_{1}\left\{\sin \left[\varphi_{0}+\left(e V_{\mathrm{H}} / \hbar\right) t\right]+(e v / \omega) \sin \omega t \cos \left[\varphi_{0}+\left(e V_{\mathrm{H}} / \hbar\right) t\right]\right\}
$$

The first term on the right-hand side self-averages to zero and the second tcrm will be nonzero whenever the AC voltage has the frequency

$$
\omega=\gamma\left(e V_{\mathrm{H}} / \hbar\right)
$$

where $\gamma$ is an integer or a fraction thereof [11]; and $\omega$ is in the microwave region. Another alternative way of detecting $J$ can be to make the system current in the $x$ direction an AC of frequency $\left(e V_{H} / \hbar\right)$. This will make the time average of $J$ non-7.ero. Whenever the average

\footnotetext{
${ }^{\dagger}$ On an uneven surface (or potential) the percolation is necessanly established first in the shortest dircction-along the width in our sample Also, the percolation establıshed in one direction excludes (or discourages) percolation in the other direction. This suits the conditions for the onset of the phase-dnven AC in the present case, in that it helps in keeping the bulk current low. I am grateful to $S$ Luryı for the communication that added to my understanding of the role played by percolation in QHE.
} 
AC between the edges becomes non-zero it alters the electric field along the sample which in tum changes the net DC flowing along the sample length (recall the relations between the components of resistivity and conductivity tensors). This effect should be observed in the form of 'Shapiro steps' [12] in the I-V characteristics.

To get an idea of the magnitude of the effect, we need to know the coupling constant $\boldsymbol{K}$ which we attempt to estimate. We can view the region separating the edge currents as a barrier running across the length of the sample. The transmittance through the barrier can be evaluated in a manner analogous to that given by Streda and co-workers [13]. To combine the quantum Hall conductance with the Landauer formula [7] they [13] consider an elastic scattering region that blocks the width of a two-dimensional sample of finite width, and study the transmittance and reflectance of the current-carrying electrons moving along the edges. Except for the difference in our case that the barrier does not block the whole width of the sample, our situation is analogous to theirs. For an electron moving along a particular edge, if $F$ represents the probability of forward transmission and $L$ represents the probability of leakage through the barrier to the other side, then following the method of [13] we obtain

$$
L=\rho_{x x} /\left(\rho_{x x}+\rho_{x y}\right)
$$

where $\rho_{x x}$ and $\rho_{x y}$ respectively represent the magnetoresistivity and the Hall resistivity.

The coupling constant $K$ will be proportional to $L$, so it is not difficult to do an orderof-magnitude calculation of $E_{c}$ and the AC. The experimental results on small devices [14] clearly indicate that $\rho_{x x}$ increases as the width of the sample decreases which according to the above and [14] can be attributed to the change in chemical potential $\mu$ along the two edges by the leakage of the electrons between them. The experimental results [14] on $\rho_{x x}$ and $\rho_{x y}$ for devices $0.25 \times \mu \mathrm{m}^{2}$ in size indicate that $L$ can be around 0.05 in the high-magnetic-field region (near plateaus 2 and 3 ) and rises by $20 \%$ or so in going to feilds in the region of plateaus 5 and 6 . Thus, about $5 \%$ of the current-carrying electrons/band can leak from one side to the other. If, to start with, we assume the variation of $\mu$ to be linear between the edges, then the lowering of the energy by $E_{T}$ can be viewed as if $\mu$ has levelled off locally over a region of width $\delta$ in the middle (where the coupling is established). Now as $\varphi$ changes by $\pi / 2$ certain electrons cross over from one side of the $\delta$-width to the other such that the energy is raised by $E_{t}$ and the original monotonic linear variation of $\mu$ is restored. For $5 \%$ of the electrons to cross over for this, the chemical potential difference will rise by $5 \%$ and so $E_{c}$ will be $0.05\left(\mu_{2}-\mu_{1}\right)$; in other words, the movement of these electrons produces a potential difference $=0.05 V_{\mathrm{H}}$ across the $\delta$-width. If we consider the vicinity of plateaus 2 and 3 where $V_{\mathrm{H}}$ is $\simeq 12 \times 10^{-6} \mathrm{~V}$ (for system current $\sim 1 \mathrm{nA}$ ) and take $\rho_{w w}=\rho_{x x} \simeq 1000 \Omega$ [14], we will get a value of $J_{y}$ of about $0.5 \mathrm{nA}$. The $J_{y}$ can be increased by about two orders of magnitude if the system current is increased by the same amount (which does not produce significant heating[14]). Thus, for $J_{c}$ in the range of $10^{-7}-10^{-9} \mathrm{~A}$, the Shapiro steps will be observable. It should be easier to observe the AC effect in very narrow samples $(<0.25 \mu \mathrm{m})$ 
at sufficiently low $V_{H}$. Indeed, there seems to be evidence for it in recent experiments by Roukes and co-workers [16] (which we will discuss in a future paper) but they are not in the quantum Hall regime.

In summary, we predict that there is yet another hidden surprise in the QHE regime which should reveal itself in the conventional Hall geometry when the sample width is reduced until it is of the order of the phase coherence length and a high-frequency (microwave) AC voltage is added to the Hall voltage. In this regime, the Hall sample begins to behave like a Josephson junction. In view of the discussion given at the beginning of the paper, it is clear that the phase-driven AC must play an inevitable role in the quantuminterference effects at 'mesoscopic' length scales [6].

Discussions with B D Josephson, M Pepper, Y Imry and R W Godby are gratefully acknowledged. Special thanks are due to Imry for pointing out the relevance of [13] in estimating the leakage between the edge currents. This work was supported by the Association of Commonwealth Universities, the University Grants Commission (New Delhi) and the University of Hyderabad.

\section{References}

[1] von Klitzing K, Dorda G and Pepper M 1980 Phvs. Rev. Lett. 45494

[2] Imry Y 1982 J.Phys C. Solid State Phys. 15 L.221: 1983 J Phys C: Solid State Phys 16 350I; 1988 Preprint of Lindel PE and Hansen O P 1983 J Phys C' : Solid State Phys 16 LI185, Tao R and Widam A 1986 Phys. Lelt 117A 481

[3] MacDonald A H and Streda P 1984 Phys Rev B29 1616

[4] Heinonen $O$ and Taylor PL 1985 Phys Rev. B32 633

[5] Laughlin R B 1981 Phys. Rev. B23

16] Washburn S and Webb R A 1986 Adv Phys 35412

[7] Büttiker M 1986 Phys. Rev. B33 3020, Landauer R 1970 Phal Mag. 21863

[8] Anderson P W 1984 Basic Notuons of Condensed Matter Physics (London Benjamin/Cammings) ch 2

[9] Feynman R P 1965 The Feynman Lectures on Physics vol III (Massachusetts : Addison-Wesley)

[10] Josephson B D 1962 Phys. Lelt 1251

[II] Dayem A H and Wiegand J 1967 Phys Rev 155419

〔12] Shapiro S 1963 Phys. Rev. Lett. 1180

[13] Streda P, Kucera J and MacDonald A H 1987 Phys Rev Lett. 59197.3

[14] Ford C J B private communication

[15] Roukes M L, Scherer A. Allen S J Jr, Craighead H G, Ruthen R M, Beebe E D and Harbison J P 1987 Phys. Rev. Lett. 593011 